\title{
AT Hook Motif
}

National Cancer Institute

\section{Source}

National Cancer Institute. AT Hook Motif. NCI Thesaurus. Code C13741.

Highly conserved peptide motifs of the HMG-I(Y) proteins that preferentially bind to the AT-rich minor groove of DNA. The AT hook motif tethers and unwinds the minor groove of the DNA with very little specificity for the DNA sequence. The AT hook motif has a narrow DNA recognition surface that is devoid of hydrophobic amino acids and that does not significantly distort the B-form DNA structure. 